Annals of Pure and Applied Mathematics

Vol. 14, No. 2, 2017, 263-268

ISSN: 2279-087X (P), 2279-0888(online)

Published on 8 September 2017

www.researchmathsci.org

DOI: http://dx.doi.org/10.22457/apam.v14n2a9

Annals of

Pure and Applied

Mathematics

\title{
Certain Topological Indices and their Polynomials of Dendrimer Nanostars
}

\author{
V.R.Kulli \\ Department of Mathematics \\ Gulbarga University, Gulbarga 585106, India \\ e-mail: vrkulli@gmail.com
}

Received 30 August 2017; accepted 8 September 2017

Abstract. Chemical graph theory is a branch of graph theory whose focus of interest is to finding topological indices of chemical graphs, which correlate well with chemical properties of the chemical molecules. In this paper, we introduce several new Zagreb polynomials of a molecular graph and compute their polynomials of dendrimer nanostars.

Keywords: Zagreb polynomial, $F$-polynomial, hyper-Zagreb polynomial, $K$-edge polynomial, reformulated Zagreb polynomial, dendrimer nanostar.

AMS Mathematics Subject Classification (2010): 05C05, 05C07, 05 C35

\section{Introduction}

Let $G$ be a connected graph. The degree $d_{G}(v)$ of a vertex $v$ is the number of vertices adjacent to $v$. The edge connecting the vertices $u$ and $v$ will be denoted by $u v$. Let $d_{G}(e)$ denote the degree of an edge $e$ in $G$, which is defined by $d_{G}(e)=d_{G}(u)+d_{G}(v)-2$ with $e$ $=u v$. For all further notation and terminology we refer to reader to [1].

A molecular graph is a simple graph related to the structure of a chemical compound. Chemical graph theory is a branch of graph theory which has an important effect on the development of the chemical sciences. A topological index is a numeric quantity from the structure of a molecule. There are several topological descriptors that have applications in Theoretical Chemistry, especially in QSPR/QSAR research see [2].

In [3], the first and second Zagreb indices of a graph $G$ are defined as

$$
\begin{aligned}
& M_{1}(G)=\sum_{u \in V(G)} d_{G}(u)^{2} \text { or } \quad M_{1}(G)=\sum_{u v \in E(G)}\left[d_{G}(u)+d_{G}(v)\right] \\
& M_{2}(G)=\sum_{u v \in E(G)} d_{G}(u) d_{G}(v) .
\end{aligned}
$$

Considering Zagreb indices, Fath-Tabar defined first and second Zagreb polynomials [4] of a graph $G$ as

$$
M_{1}(G, x)=\sum_{u v \in E(G)} x^{d_{G}(u)+d_{G}(v)} \quad \text { or } \quad M_{2}(G, x)=\sum_{u v \in E(G)} x^{d_{G}(u) d_{G}(v)}
$$



graph $G$ as

Considering another first Zagreb index, we define vertex Zagreb polynomial of a

$$
M_{v}(G, x)=\sum_{u \in V(G)} x^{d_{G}(u)^{2}} .
$$

In [5], Furtula and Gutman studied the forgotten topological index or $F$-index and defined it as

$$
F(G)=\sum_{u \in V(G)} d_{G}(u)^{3} \quad \text { or } \quad F(G)=\sum_{u v E(G)}\left[d_{G}(u)^{2}+d_{G}(v)^{2}\right]
$$

In [6], De et al. defined $F$-polynomial of a graph $G$ as

$$
F(G, x)=\sum_{u v \in E(G)} x^{d_{G}(u)^{2}+d_{G}(v)^{2}} .
$$

Also considering another $F$-index, we define vertex $F$-polynomial of a graph $G$ as

$$
F_{1}(G, x)=\sum_{u \in V(G)} x^{d_{G}(u)^{3}} .
$$

In [7], Chaluvaraju et al. defined first and second hyper-Zagreb polynomials as

$$
H M_{1}(G, x)=\sum_{u v \in E(G)} x^{\left[d_{G}(u)+d_{G}(v)\right]^{2}}, H M_{2}(G, x)=\sum_{u v \in E(G)} x^{\left[d_{G}(u) d_{G}(v)\right]^{2}} .
$$

In [8], Kulli defined general first and second Zagreb polynomails as

$$
M_{1}^{a}(G, x)=\sum_{u v E(G)} x^{\left[d_{G}(u)+d_{G}(v)\right]^{a}}, \quad M_{2}^{a}(G, x)=\sum_{u v E E(G)} x^{\left[d_{G}(u) d_{G}(v)\right]^{a}}
$$

where $a$ is a real number.

In [9], Miličević et al. introduced the reformulated first and second Zagreb indices of a graph $G$ and they are defined as

$$
E M_{1}(G)=\sum_{e \in E(G)} d_{G}(e)^{2} ., \quad E M_{2}(G)=\sum_{e \sim f} d_{G}(e) d_{G}(f) .
$$

Considering the reformulated Zagreb indices, we define reformulated first and second Zagreb polynomials as

$$
E M_{1}(G, x)=\sum_{e \in E(G)} x^{d_{G}(e)^{2}}, \quad E M_{2}(G, x)=\sum_{e \sim f} x^{d_{G}(e) d_{G}(f)} .
$$

where $e \sim f$ means that the edges $e, f$ are adjacent.

In [10], Kulli introduced the $K$-edge index of a graph $G$ and defined it as

$$
K_{e}(G)=\sum_{e \in E(G)} d_{G}(e)^{3} \text {. }
$$

Considering the $K$-edge index, we define the $K$-edge polynomial as

$$
K_{e}(G, x)=\sum_{e \in E(G)} x^{d_{G}(e)^{3}} .
$$

We introduce another polynomial related to the first reformulated Zagreb index as

$$
E M_{0}(G, x)=\sum_{e \in E(G)} x^{d_{G}(e)} .
$$


Certain Topological Indices and their Polynomials of Dendrimer Nanostars

Also we define the general reformulated first and second Zagreb polynomials as

$$
E M_{1}^{a}(G, x)=\sum_{e \in E(G)} x^{d_{G}(e)^{a}}, \quad E M_{2}^{a}(G, x)=\sum_{e \sim f} x^{\left[d_{G}(e) d_{G}(f)\right]^{a}} .
$$

Recently, some topological indices were studied in $[11,12,13]$.

In this paper, certain Zagreb polynomials of dendrimer nanostars are determined. For dendrimer nanostars $D_{3}[n]$ see [14] and references cited therein.

\section{Results}

We consider the dendrimer nanostar with $n$ growth stages, denoted by $D_{3}[n]$, where $n \geq 0$, see Figure 1. Let $G$ be the dendrimer nanostar $D_{3}[n]$. From Figure 1, it is easy to see that the vertices of $D_{3}[n]$ are either of degree 1,2 or 3 . By calculation, we obtain that $D_{3}[n]$ has $24 \times 2^{n}-20$ vertices and $24\left(2^{n+1}-1\right)$ edges.

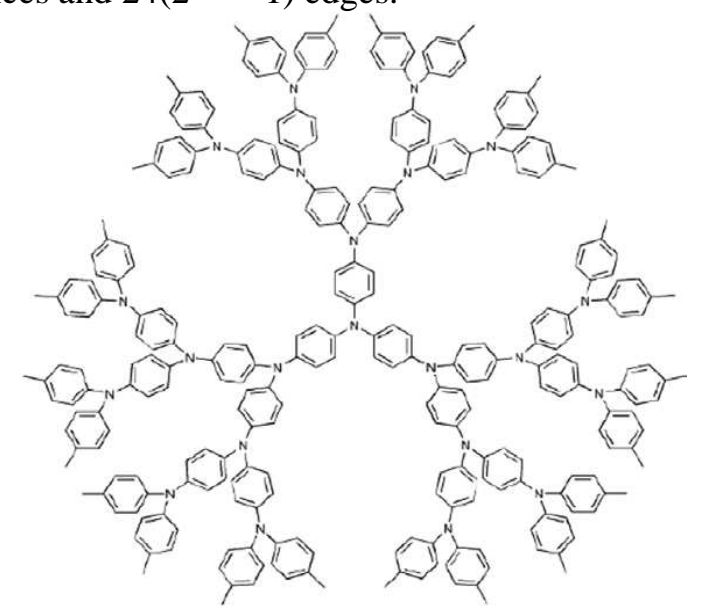

Figure1: Dendrimer nanostar with 3-growth $D_{3}[n]$

We partition $V(G)$ into three sets, vertices of degree 1, 2 and 3 respectively.

$$
\begin{array}{llrl}
V_{1} & =\left\{u \in V(G) \mid d_{G}(u)=1\right\}, & & \left|V_{1}\right|=3 \times 2^{n} . \\
V_{2} & =\left\{u \in V(G) \mid d_{G}(u)=2\right\}, & & \left|V_{2}\right|=6 \times 2^{n}-12 . \\
V_{3} & =\left\{u \in V(G) \mid d_{G}(u)=3\right\}, & & \left|V_{3}\right|=15 \times 2^{n}-8 .
\end{array}
$$

Also by algebraic method, we partition $E(G)$ into four sets, based on the sum of degrees of the end vertices of each edge.

$$
\begin{array}{ll}
E_{13}=\left\{u v \in E(G) \mid d_{G}(u)=1, d_{G}(v)=3\right\}, & \left|E_{13}\right|=3 \times 2^{n} . \\
E_{22}=\left\{u v \in E(G) \mid d_{G}(u)=d_{G}(v)=2\right\}, & \left|E_{22}\right|=12 \times 2^{n}-6 . \\
E_{23}=\left\{u v \in E(G) \mid d_{G}(u)=2, d_{G}(v)=3\right\}, & \left|E_{23}\right|=24 \times 2^{n}-12 . \\
E_{33}=\left\{u v \in E(G) \mid d_{G}(u)=d_{G}(v)=3\right\}, & \left|E_{33}\right|=9 \times 2^{n}-6 .
\end{array}
$$

The edge degree partition of $G$ is given in Table 1 .

\begin{tabular}{cccc}
\hline$d_{G}(u) d_{G}(v) \backslash u v \in E(G)$ & $E_{4}=(1,3) \cup(2,2)$ & $E_{5}=(2,3)$ & $E_{6}=(3,3)$ \\
\hline$d_{G}(e)$ & 2 & 3 & 4 \\
\hline Number of edges & $15 \times 2^{n}-6$ & $24 \times 2^{n}-12$ & $9 \times 2^{n}-6$ \\
\hline
\end{tabular}

Table 1: Edge degree partition of $G$. 
Theorem 1. The vertex Zagreb polynomial of a dendrimer nanostar $D_{3}[n]$ is

$$
M_{v}\left(D_{3}[n], x\right)=\left(15 \times 2^{n}-8\right) x^{9}+\left(6 \times 2^{n}-12\right) x^{4}+3 \times 2^{n} x
$$

Proof: For the vertex Zagreb polynomial of $D_{3}[n]$, we have

$$
\begin{aligned}
& M_{v}\left(D_{3}[n], x\right)=\sum_{u \in V} x^{d_{G}(u)^{2}}=\sum_{V_{1}} x^{d_{G}(u)^{2}}+\sum_{V_{2}} x^{d_{G}(u)^{2}}+\sum_{V_{3}} x^{d_{G}(u)^{2}} \\
& =3 \times 2^{n} x^{1^{2}}+\left(6 \times 2^{n}-12\right) x^{2^{2}}+\left(15 \times 2^{n}-8\right) x^{3^{2}} \\
& =\left(15 \times 2^{n}-8\right) x^{9}+\left(6 \times 2^{n}-12\right) x^{4}+3 \times 2^{n} x
\end{aligned}
$$

Theorem 2. The vertex $F$-polynomial of a dendrimer nanostar $D_{3}[n]$ is

$$
F_{v}\left(D_{3}[n], x\right)=\left(15 \times 2^{n}-8\right) x^{27}+\left(6 \times 2^{n}-12\right) x^{8}+3 \times 2^{n} x .
$$

Proof: For the vertex $F$-polynomial of a dendrimer nanostar $D_{3}[n]$ is

$$
\begin{aligned}
& F_{v}\left(D_{3}[n], x\right)=\sum_{u \in V} x^{d_{G}(u)^{3}}=\sum_{V_{3}} x^{d_{G}(u)^{3}}+\sum_{V_{2}} x^{d_{G}(u)^{3}}+\sum_{V_{1}} x^{d_{G}(u)^{3}} \\
& =\left(15 \times 2^{n}-8\right) x^{3^{3}}+\left(6 \times 2^{n}-12\right) x^{2^{3}}+3 \times 2^{n} x^{1^{3}} . \\
& =\left(15 \times 2^{n}-8\right) x^{27}+\left(6 \times 2^{n}-12\right) x^{8}+3 \times 2^{n} x
\end{aligned}
$$

Theorem 3. The $F$-polynomial of a dendrimer nanostar $D_{3}[n]$ is

$$
F\left(D_{3}[n], x\right)=3 \times 2^{n} x^{10}+\left(12 \times 2^{n}-6\right) x^{8}+\left(24 \times 2^{n}-12\right) x^{13}+\left(9 \times 2^{n}-6\right) x^{18} \text {. }
$$

Proof: For the $F$-polynomial of a dendrimer nanostar $D_{3}[n]$ is

$$
\begin{aligned}
& F\left(D_{3}[n], x\right)=\sum_{u v \in E} x^{d_{G}(u)^{2}+d_{G}(v)^{2}} \\
& =\sum_{E_{13}} x^{d_{G}(u)^{2}+d_{G}(v)^{2}}+\sum_{E_{22}} x^{d_{G}(u)^{2}+d_{G}(v)^{2}}+\sum_{E_{23}} x^{d_{G}(u)^{2}+d_{G}(v)^{2}}+\sum_{E_{33}} x^{d_{G}(u)^{2}+d_{G}(v)^{2}} \\
& =3 \times 2^{n} x^{10}+\left(12 \times 2^{n}-6\right) x^{8}+\left(24 \times 2^{n}-12\right) x^{13}+\left(9 \times 2^{n}-6\right) x^{18} .
\end{aligned}
$$

Theorem 4. The general first Zagreb polynomial of a dendrimer nanostar $D_{3}[n]$ is $M_{1}^{a}\left(D_{3}[n], x\right)=\left(15 \times 2^{n}-6\right) x^{4^{a}}+\left(24 \times 2^{n}-12\right) x^{5^{a}}+\left(9 \times 2^{n}-6\right) x^{6^{a}}$.

Proof: For the general first Zagreb polynomial of a dendrimer nanostar $D_{3}[n]$, we have

$$
\begin{aligned}
& M_{1}^{a}\left(D_{3}[n], x\right)=\sum_{u v \in E} x^{\left[d_{G}(u)+d_{G}(v)\right]^{a}} \\
& =\sum_{E_{13}} x^{\left[d_{G}(u)+d_{G}(v)\right]^{a}}+\sum_{E_{22}} x^{\left[d_{G}(u)+d_{G}(v)\right]^{a}}+\sum_{E_{23}} x^{\left[d_{G}(u)+d_{G}(v)\right]^{a}}+\sum_{E_{33}} x^{\left[d_{G}(u)+d_{G}(v)\right]^{a}} \\
& =3 \times 2^{n} x^{4^{a}}+\left(12 \times 2^{n}-6\right) x^{4^{a}}+\left(24 \times 2^{n}-12\right) x^{5^{a}}+\left(9 \times 2^{n}-6\right) x^{6^{a}} .
\end{aligned}
$$

Corollary 4.1. The first Zagreb polynomial of $D_{3}[n]$ is

$$
M_{1}\left(D_{3}[n], x\right)=M_{1}^{1}\left(D_{3}[n], x\right)=\left(15 \times 2^{n}-6\right) x^{4}+\left(24 \times 2^{n}-12\right) x^{5}+\left(9 \times 2^{n}-6\right) x^{6} \text {. }
$$


Certain Topological Indices and their Polynomials of Dendrimer Nanostars

Corollary 4.2. The first hyper-Zagreb polynomial of $D_{3}[n]$ is

$H M_{1}\left(D_{3}[n], x\right)=M_{1}^{2}\left(D_{3}[n], x\right)=\left(15 \times 2^{n}-6\right) x^{16}+\left(24 \times 2^{n}-12\right) x^{25}+\left(9 \times 2^{n}-6\right) x^{36}$.

Theorem 5. The general second Zagreb polynomial of a dendrimer nanostar $D_{3}[n]$ is $M_{2}^{a}\left(D_{3}[n], x\right)=3 \times 2^{n} x^{3^{a}}+\left(12 \times 2^{n}-6\right) x^{4^{a}}+\left(24 \times 2^{n}-12\right) x^{6^{a}}+\left(9 \times 2^{n}-6\right) x^{9^{a}}$.

Proof: For the general second Zagreb polynomial of a dendrimer nanostar $D_{3}[n]$, we have

$$
\begin{aligned}
& M_{2}^{a}\left(D_{3}[n], x\right)=\sum_{E} x^{\left[d_{G}(u) d_{G}(v)\right]^{a}} \\
& =\sum_{E_{13}} x^{\left[d_{G}(u) d_{G}(v)\right]^{a}}+\sum_{E_{22}} x^{\left[d_{G}(u) d_{G}(v)\right]^{a}}+\sum_{E_{23}} x^{\left[d_{G}(u) d_{G}(v)\right]^{a}}+\sum_{E_{33}} x^{\left[d_{G}(u) d_{G}(v)\right]^{a}} \\
& =3 \times 2^{n} x^{3^{a}}+\left(12 \times 2^{n}-6\right) x^{4^{a}}+\left(24 \times 2^{n}-12\right) x^{6^{a}}+\left(9 \times 2^{n}-6\right) x^{a^{a}} .
\end{aligned}
$$

Corollary 5.1. The second Zagreb polynomial of $D_{3}[n]$ is

$M_{2}\left(D_{3}[n], x\right)=M_{2}^{1}\left(D_{3}[n], x\right)=3 \times 2^{n} x^{3}+\left(12 \times 2^{n}-6\right) x^{4}+\left(24 \times 2^{n}-12\right) x^{6}+\left(9 \times 2^{n}-6\right) x^{9}$.

Corollary 5.2. The second hyper-Zagreb polynomial of $D_{3}[n]$ is

$H M_{2}\left(D_{3}[n], x\right)=M_{2}^{2}\left(D_{3}[n], x\right)=3 \times 2^{n} x^{9}+\left(12 \times 2^{n}-6\right) x^{16}+\left(24 \times 2^{n}-12\right) x^{36}+\left(9 \times 2^{n}-6\right) x^{81}$.

Theorem 6. The general reformulated first Zagreb polynomial of a dendrimer nanostar $D_{3}[n]$ is

$$
\operatorname{EM}_{1}^{a}\left(D_{3}[n], x\right)=\left(15 \times 2^{n}-6\right) x^{2^{a}}+\left(24 \times 2^{n}-12\right) x^{3^{a}}+\left(9 \times 2^{n}-6\right) x^{4^{a}} \text {. }
$$

Proof: For the general reformulated first Zagreb polynomial of a dendrimer nanostar $D_{3}[n]$ is

$$
\begin{aligned}
& E M_{1}^{a}\left(D_{3}[n], x\right)=\sum_{e \in E(G)} x^{d_{G}(e)^{a}} \\
& =\sum_{e \in E_{4}} x^{d_{G}(e)^{a}}+\sum_{e \in E_{5}} x^{d_{G}(e)^{a}}+\sum_{e \in E_{6}} x^{d_{G}(e)^{a}} \\
& =\left(15 \times 2^{n}-6\right) x^{2^{a}}+\left(24 \times 2^{n}-12\right) x^{3^{a}}+\left(9 \times 2^{n}-6\right) x^{4^{a}} .
\end{aligned}
$$

Corollary 6.1. The reformulated Zagreb polynomial of $D_{3}[n]$ is $E M_{0}\left(D_{3}[n], x\right)=E M_{1}^{1}\left(D_{3}[n], x\right)=\left(15 \times 2^{n}-6\right) x^{2}+\left(24 \times 2^{n}-12\right) x^{3}+\left(9 \times 2^{n}-6\right) x^{4}$.

Corollary 6.2. The reformulated first Zagreb polynomial of $D_{3}[n]$ is $E M_{1}\left(D_{3}[n], x\right)=E M_{1}^{2}\left(D_{3}[n], x\right)=\left(15 \times 2^{n}-6\right) x^{4}+\left(24 \times 2^{n}-12\right) x^{9}+\left(9 \times 2^{n}-6\right) x^{16}$.

Corollary 6.3. The $K$-edge polynomial of $D_{3}[n]$ is

$$
K_{e}\left(D_{3}[n], x\right)=E M_{1}^{3}\left(D_{3}[n], x\right)=\left(15 \times 2^{n}-6\right) x^{8}+\left(24 \times 2^{n}-12\right) x^{27}+\left(9 \times 2^{n}-6\right) x^{64} \text {. }
$$




\section{V.R.Kulli}

\section{REFERENCES}

1. V.R.Kulli, College Graph Theory, Vishwa International Publications, Gulbarga, India (2012).

2. I.Gutman and O.E.Polansky, Mathematical Concepts in Organic Chemistry, Springer, Berlin, (1986).

3. I.Gutman and N.Trinajstić, Graph theory and molecular orbitals. Total $\square$-electron energy of alternant hydrocarbons, Chem. Phys. Lett., 17 (1972) 535-538.

4. G.H.Fath-Tabar, Zagreb polynomial and pi indices of some nanostructures, Digest Journal of Nanomaterials and Biostructures, 4(1) (2009) 189-191.

5. B.Furtula and I.Gutman, A forgotten topological index, J. Math. Chem. 53 (2015), 1184-1190.

6. N.De and S.M.A.Nayeem, Computing the $F$-index of nanostar dendrimers, Pacific Science Review A: Natural Science and Engineering, (2016) DOI:http:/dx.doi.org/ 10.1016/j.psra.2016.06.001.

7. B.Chaluvaraju, H.S.Boregowda and S.A. Diwakar, Hyper-Zagreb indices and their polynomials of some special kinds of windmill graphs, International Journal of Advances in Mathematics, 4 (2017) 21-32

8. V.R.Kulli, General Zagreb polynomials and $F$-polynomial of certain nanostructrues, submitted.

9. A.Miličević, S.Nikolić and N.Trinajstić, On reformulated Zagreb indices, Mol. Divers., 8 (2004) 393-399.

10. V.R.Kulli, On $K$ - edge index and coindex of graphs, International Journal of Fuzzy Mathematical Archive, 10(2) (2016) 111-116.

11. V.R.Kulli, New arithmetic-geometric indices, Annals of Pure and Applied Mathematics, 13(2) (2017) 165-172.

12. V.R.Kulli, The Gourava indices and coindices of graphs, Annals of Pure and Applied Mathematics, 14(1) (2017) 33-38.

13. V.R.Kulli, Two new multiplicative atom bond connectivity indices, Annals of Pure and Applied Mathematics, 13(1) (2017) 1-7.

14. V.R.Kulli, Computing topological indices of dendrimer nanostars, International Journal of Mathematics and its applications, 5(3-A) (2017) 163-169. 